\title{
IMPLEMENTACIÓN DE UNA PROPUESTA DE APRENDIZAJE SIGNIFICATIVO DE LA CINEMÁTICA A TRAVÉS DE LA RESOLUCIÓN DE PROBLEMAS
}

\section{IMPLEMENTATION OF A PROPOSAL FOR MEANINGFUL LEARNING OF KINEMATICS THROUGH PROBLEM SOLVING}

\author{
Iván Sánchez Soto ${ }^{1} \quad$ Marco Antonio Moreira ${ }^{2} \quad$ Concesa Caballero Sahelices ${ }^{3}$ \\ Recibido 21 de agosto de 2007, aceptado 13 de noviembre de 2008 \\ Received: August 21, 2007 Accepted: November 13, 2008
}

\begin{abstract}
RESUMEN
El presente trabajo tiene por finalidad compartir las implicaciones didácticas que surgen del diseño y la aplicación de una propuesta metodológica activa, basada en la resolución de problemas y uso de cálculo diferencial, como medios para abordar los contenidos de cinemática, con la intención de facilitar y promover la adquisición de aprendizaje significativo de conceptos, procedimientos, actitudes, principios y leyes que rigen la cinemática dentro de la asignatura Física I, dictada para estudiantes de Ingeniería de la Universidad del Bío-Bío, Chile. El punto de partida de la propuesta consiste en presentar un problema integrador de los contenidos de cinemática que sirve de columna vertebral de la unidad programática a aprender, y que a su vez se puede dividir en una serie de problemas más acotados para abordar los diferentes contenidos de la unidad. Los resultados alcanzados muestran una valoración favorable de los estudiantes y han permitido establecer la influencia de la propuesta metodológica en el rendimiento académico y estrategias de aprendizaje, como indicadores de aprendizaje significativo y del reconocimiento que el alumno atribuye a la propuesta.
\end{abstract}

Palabras clave: Aprendizaje significativo, problemas integrador, metodología activo-participativa.

\begin{abstract}
This paper aims at sharing some pedagogical implications that emerge from the design and application of an active methodological proposal based on problem solving and on the use of differential calculus as a means to approach the contents of kinematics to promote and facilitate meaningful learning of concepts, procedures, attitudes, principles, and laws that govern kinematics in the course of Physics I for students of engineering at the Universidad del Bío-Bio, Chile. The starting point of this proposal consists of presenting an integrating problem for the contents of kinematics, which are at the very core of the learning unit, and that, in turn, can be divided into a series of more localized problems to deal with the different/various contents of this unit. Research findings suggest that students present a favorable opinion about this proposal, and that these findings seem to point out an influence of the methodological proposal on academic achievement and learning strategies as indicators of meaningful learning as well as the value assigned by the students to this proposal.
\end{abstract}

Keywords: Meaningful learning, integrating problem, learning strategies.

\section{INTRODUCCIÓN}

En el presente trabajo se pretende mostrar las implicaciones didácticas de una propuesta metodológica activa, que busca facilitar la adquisición de un aprendizaje significativo a través de la resolución de problemas (ASARP), que incluyen actividades de aprendizaje (A.A) y uso del cálculo diferencial para resolver los ejercicios y ejemplos presentados en el aula, y así poner de manifiesto la importancia de los conceptos involucrados en la solución (condiciones físicas iniciales y finales) y la evaluación de los conceptos físicos involucrados [1]. Esta forma de abordar contenidos lleva a describir e interpretar los problemas y ejercicios planteados a través de un modelo único, que induce una forma similar de resolver casi todos los ejercicios de cinemática, rescatando la interpretación física del problema y no el uso de fórmulas a memorizar [1]. De esta manera, se pretende evidenciar que los

\footnotetext{
1 Universidad del Bío-Bío. Concepción, Chile. E-mail: isanchez@ubiobio.cl

2 Instituto de Física. Universidade Federal do Rio Grande do Sul (UFRGS). Porto Alegre, Brasil. E-mail: moreira@if.ufrgs.br

3 Universidad de Burgos. Burgos, España. E-mail: concesa@ubu.es
} 
contenidos de cinemática comprenden un conjunto de conceptos, principios, leyes y reglas que se deben aprender significativamente.

A través del uso del cálculo diferencial, en esta propuesta, se adquiere un único método de resolución de ejercicios y problemas cerrados, donde no se considera el uso de fórmulas, sino que se desarrolla en el alumno la habilidad de interpretar, describir y transferir los conocimientos adquiridos en nuevas situaciones, a través de la comprensión de lo que realiza. Es decir, eliminando el uso indiscriminado de fórmulas para llegar a la solución, y evitando aprender mecánicamente la asociación de cada ejercicio con una u otra fórmula. La propuesta posibilita el abandono del uso mecánico de éstas y la correspondencia entre ejercicio y fórmula.

El punto de partida de la propuesta consiste en plantear un problema integrador de contenidos, como un desafío, a resolver al final de la unidad programática por los alumnos. En primera instancia, sirve para establecer los conocimientos previos, contenidos a investigar y los posibles escenarios, a partir de los cuales se plantean una serie de problemas más acotados en contenidos y sus correspondientes AA, que llevan a la solución de cada problema, a través de los conocimientos adquiridos al resolver las AA. Esto implica que los alumnos deben considerar la situación Física en su máxima amplitud, superando la mera manipulación de las expresiones algebraicas; deben analizar, con detenimiento y críticamente, hasta lo más obvio, eliminando los ejercicios con datos y uso mecánico de fórmulas para encontrar incógnitas, lo que resulta de gran importancia para la construcción del conocimiento.

La propuesta hace uso de una diversidad de AA que incluyen ejercicios de tipos cualitativos y cuantitativos, de carácter cerrado y abierto, con la intención de verificar el uso de cálculo diferencial con comprensión y sentido físico, y así eliminar el uso mecánico de fórmulas que se adquieren de manera superficial y reiterativa. Estas AA se adaptan a la diversidad de alumnos y se presentan con una complejidad gradual, que comprende exploración de ideas previas, introducción de variables, síntesis y transferencia de contenidos a situaciones nuevas, las que se acometen con mayor o menor grado de éxito.

Se espera que el trabajo conduzca al diseño y elaboración de una propuesta metodológica activa y participativa, que aparezca como una alternativa posible para superar las limitaciones detectadas en el aprendizaje de conceptos y procedimientos propios de la Física, es decir, presentar una metodología de trabajo en el aula específica, que se cree puede tener un alto impacto en el desempeño de la docencia, el desarrollo de competencias laborales y habilidades cognitivas en los estudiantes. Se pretende extender la propuesta metodológica para el proceso de enseñar y aprender (E-A) todos los contenidos de Física General I, a partir de un problema abarcador y una serie de problemas, más acotados, entramados entre sí, que promuevan el trabajo en equipo, la interacción en el aula de la tríada material educativo, profesor y alumno, y así facilitar la abstracción y transferencia de los contenidos a situaciones nuevas, donde se procese la información de manera profunda y elaborativa, es decir, se aprenda significativamente.

Para atender los propósitos planteados se diseña y aplica una propuesta metodológica activa para el ASARP, que se caracteriza, por ser un método de trabajo activo donde los alumnos participan constantemente en la adquisición de su conocimiento. Ésta pretende promover el trabajo en grupos pequeños, orientado a la solución de problemas que son seleccionados o diseñados a partir de noticias, periódicos, etc.; donde se aprende investigando y buscando la información de los contenidos y de la propia experiencia de trabajo en el aula (dinámica del método). Las AA giran en torno a la discusión de un problema, y el aprendizaje se adquiere de la experiencia de trabajar sobre ese problema, que es asumido por el alumno y no por el profesor, el cual juega un rol de mediador, negociador de significado.

La propuesta tiene por finalidad mejorar el proceso de (E-A), favorecer y facilitar la adquisición de un aprendizaje significativo de conceptos, procedimientos, actitudes y habilidades de comunicación en la asignatura de Física I, a partir de la implementación del ASARP como metodología de trabajo en el aula, que pretende promover la adquisición del conocimiento en un contexto metodológico activo-participativo, y así establecer su influencia en las estrategias de aprendizaje y rendimiento académico de los alumnos.

A través de la propuesta de aprendizaje con base en problemas, como metodología para enseñar y aprender, se busca superar algunas dificultades para captar aprendizaje significativo como son: a) Los alumnos están acostumbrados a escuchar pasivamente al profesor y memorizar, por tanto, cualquier cambio en la metodología de trabajo, provoca al principio inseguridad en los estudiantes; $b$ ) El alumno no reconoce su carencia de conocimientos, y c) La comunicación de información oral y escrita de los alumno, en general, es muy superficial.

La propuesta ha sido aplicada a estudiantes de Ingeniería en Ejecución eléctrica y electrónica de la Universidad del 
Bío-Bío (Chile), con la finalidad de: facilitar la adquisición de aprendizaje significativo, eliminar el uso mecánico e indiscriminado de fórmulas, desarrollar estrategias de aprendizaje, mejorar el rendimiento académico y valoración de la propuesta. Los resultados obtenidos en dos aplicaciones sistemáticas de la propuesta metodológica bajo ABP son alentadores; la muestra de alumnos manifestó estar motivados, valorando favorablemente la metodología, lo que presupone su validez en otros campos del saber.

\section{FUNDAMENTOS TEÓRICOS}

La presente propuesta metodológica de innovación ASARP implica plantear problemas a resolver en la clase (aula), capaces de promover: la búsqueda de información, la investigación orientada, el trabajo en equipo abordando los contenidos del curso a partir de una situación real, y así facilitar la interacción entre lo que los alumnos conocen y lo que deben aprender, condición necesaria para el aprendizaje significativo.

Se pretende, por medio de la propuesta, que los alumnos aprendan conceptos (saber), procedimientos (saber hacer), actitudes (saber ser), leyes y principios propios de la cinemática y que desarrollen estrategias de aprendizaje, profundas y elaborativas, dentro del programa del curso [2]. Esto nos sitúa ante la misma práctica del docente, como generador de un sistema Metodológico-Interactivo, quien debe configurar un sistema metodológico propio, un estilo Socio-Comunicativo, que armonice una cultura plenamente universitaria con las vivencias del saber; es decir, un modo creador de conocimiento y de transformación profesional. Por otra parte, "la acción docente y mejora didáctica debe considerar: Acompañar al estudiante, colaborar en la construcción de conocimiento y aprendizaje significativo, interpretar el sentido de la formación desde cada participante, valorar el proceso y no sólo resultados" [3].

Desde esta perspectiva, la propuesta metodológica con base a un problema integrador y una secuencia de problemas más acotados implica incluir una secuencia de AA que comprenden según [4-5] las fases de: exploración de ideas, introducción de variables, síntesis y transferencia de contenidos a situaciones nuevas, simples y complejas, para interpretar la realidad.

Cabe destacar que el aprendizaje de la Física tiene la particularidad de requerir el empleo de las operaciones mentales de mayor complejidad. Es decir, la apropiación de los conocimientos debe evidenciarse mediante procedimientos tales como la resolución de problemas.
Algunos autores conciben la resolución de problemas como un proceso que reproduce procedimientos de la investigación científica [6-8]. "El éxito de la resolución de problemas depende de distintas variables que afectan al problema en sí, al estudiante, al profesor y al contexto de la resolución" [5]. Estas técnicas, con sus limitaciones, constituyen una aproximación al trabajo científico, promoviendo sus aplicaciones prácticas y la transferencia de los conocimientos a lo cotidiano.

La crítica al proceso enseñar y aprender (E-A) por descubrimiento autónomo e inductivo, donde se valora el método sobre el contenido y no se considera el papel de la interacción social [5]. Esto es imposible ya que los procesos de las ciencias no se presentan separados de los contenidos, y el desarrollo de las destrezas intelectuales se produce sobre campos conceptuales concretos [5]. De aquí se plantea una nueva forma de E-A, dando importancia a los conocimientos previos, que el alumno dispone y a la integración de los nuevos, promoviendo un desarrollo cognitivo. Se resalta así la guía del profesor, como el orientador y mediador del aprendizaje significativo [9], quien activa el proceso de asimilación en la construcción de conocimientos [6] con un tiempo propio para la reflexión y la crítica.

La propuesta metodológica, diseñada y aplicada (ASARP), se sustenta en diferentes corrientes teóricas sobre el aprendizaje humano, como son la teoría del aprendizaje significativo de [10-11], el modelo de enseñanza aprendizaje (tríada profesor, alumnos y materiales de enseñanza para negociar significado) propuesta por [12]. Estas teorías forman un cuerpo teórico coherente sobre el proceso de E-A en el aula. También se considera y se destaca la teoría de la interacción social (el aprendizaje se genera en las zonas del desarrollo próximo) de [13]. En estas teorías, en conjunto, tiene particular presencia la teoría constructivista; de acuerdo con esta postura, en el diseño, elaboración y aplicación del ASARP, es de gran importancia el contexto y presentación del problema para lograr un aprendizaje significativo [14].

En particular, el ASARP se sustenta en la "teoría del aprendizaje significativo de David Ausubel, quien supuso el primer modelo sistemático de aprendizaje cognitivo" [16-17], donde el concepto central es el de aprendizaje significativo, proceso a través del cual una misma información se relaciona, de manera no arbitraria y sustancial (no al pie de la letra), con lo que el alumno ya sabe, y si éste adopta la actitud de aprendizaje correspondiente para hacerlo así [16]. Es decir, para que el aprendizaje sea significativo, el material empleado para enseñar y aprender debe ser potencialmente significativo y el alumno debe 
manifestar una disposición para aprender, y así la nueva información entra en interacción con una estructura de conocimiento específica (concepto subsumidor) existente en la estructura cognitiva de quien aprende.

En contraposición con el aprendizaje significativo [16] define aprendizaje mecánico o superficial y reiterativo, estableciendo diferencias que tienen lugar cuando el estudiante no relaciona la nueva información con la existente en su estructura cognitiva. De manera que los nuevos conocimientos se aprenden de forma aislada y sin relación entre sí, por lo que no contribuyen al aprendizaje significativo y, más bien, lo dificultan. Según [16], entre estos dos extremos existiría un continuo que permitiría encuadrar la mayoría de las situaciones de aprendizaje de los estudiantes. La distinción entre aprendizaje significativo y aprendizaje memorístico es independiente de que éste se lleve a cabo por recepción o por descubrimiento. Para [16], la distinción entre significativo y mecánico no establece una dicotomía, sino un continuo. Por ejemplo, la simple memorización de fórmulas se situaría en uno de los extremos de ese continuo (superficial y reiterativo), mientras que el aprendizaje de relaciones entre conceptos podría estar en el otro extremo (significativo).

\section{METODOLOGÍA DE AULA}

Los resultados previos obtenidos [1, 14], afirman que el empleo de la metodología activa, con base en problemas, favorece las prácticas pedagógicas de los académicos y apuntan hacia el logro de aprendizaje más significativo, el desarrollo de capacidades para el trabajo en equipo, la mejora de las capacidades de comunicación oral y escrita.

Si bien los entornos de aprendizaje, con base en problemas, pueden ser implementados de muchas formas en el aula, en todas ellas se encuentran características comunes, como son, la siguiente secuencia de etapas: a) Primera, de trabajo en equipo, donde se presenta un texto que describe una situación, a partir del cual se identifican el o los problemas que se presentan o derivan del texto, se identifican los contenidos que se asignan para investigar a los diferentes integrantes del equipo, y finalmente, se plantean los objetivos de aprendizaje. b) La segunda, que comprende el trabajo individual, donde los alumnos deben buscar el máximo de información e investigar acerca de los contenidos asignados por el grupo. c) En la tercera, se considera la socialización de contenidos y, en caso de tener todos los elementos, resolver el problema, solución y presentación del problema resuelto; de lo contrario, iniciar una nueva iteración hasta obtener todos los elementos necesarios [18].
La propuesta de aprendizaje con base en un problema integrador y secuencia de problemas más acotados, al igual que el ABP propuestos por [19-21], tiene por finalidad que los alumnos aprendan en pequeños grupos, a partir de un problema, buscar la información necesaria para comprender el problema y obtener una solución bajo la supervisión de un tutor. Aquí se favorece el desarrollo de habilidades cognitivas y de socialización, de análisis y síntesis de la información y de actitudes positivas frente a problemas. Éstas se diferencian en que el problema integrador es el hilo o eje conductor de la unidad en estudio; los problemas acotados se estructuran en torno a esta situación, según una secuencia lógica para alcanzar la solución del problema integrador al final de la unidad. Sin embargo, para [20,22], en el ABP los problemas provienen de un contexto real (noticias), pero sin conexión entre sí.

Los contenidos de cinemática se abordan a partir de un problema abierto e integrador de contenido a resolver, que sirve de hilo conductor de la asignatura y, a partir de cual, se define una secuencia integrada de problemas más acotados en contenidos, según un criterio jerárquico, que se inicia con: parámetros de la cinemática, movimiento en una dimensión y movimiento en dos dimensiones. La solución de los problemas más acotados o específicos lleva a comprender y resolver el problema integrador, profundizando en los contenidos que son necesarios para su resolución. Por otra parte, esta forma de abordar el proceso de (E-A) permite mostrar la jerarquía de los contenidos y así eliminar su acostumbrada fragmentación a través de la diferenciación progresiva y la reconciliación integradora de los contenidos.

\section{PROPUESTA METODOLÓGICA}

A partir de la experiencia y resultados alcanzados [14, 18], se establece la siguiente secuencia de actividades para abordar los contenidos en el aula: a) La unidad programática a tratar debe ser abordada a partir del planteamiento de un problema integrador de contenidos, desde el inicio, con suficiente entidad (objeto con existencia conceptual) para estructurar la actividad del aula en torno a él; b) El problema integrador debe permitir ser abordado por diferentes problemas más pequeños e independientes entre sí; c) La secuencia de problemas más pequeños debe aportar a la solución del problema integrador; d) Los hechos, conceptos, principios, leyes y modelos se deben introducir con algún propósito o intención para lograr la solución de los problemas más acotados; e) La integración de los contenidos adquiridos en la solución de los problemas más acotados y en sus correspondientes 
actividades de aprendizaje debe ser realizada por el alumno al resolver el problema integrador.

Para [14] y [23] el programa de actividades para trabajar los problemas en el aula, considera: a) Plantear y presentar a los alumnos un problema integrador de contenidos de actualidad, en lo posible, con una carga de afectividad que sea motivador para los alumnos, promoviendo su resolución; b) Previa lectura del problema integrador, identificar los conceptos previos y contenidos a investigar, lo que lleva a plantear preguntas que orientan la investigación, en lo posible, en el contexto de la especialidad; c) Puesta en común de los conocimientos previos, trabajo colaborativo, compartiendo el significado del contenido investigado; d) Se acotan los contenidos a abordar, por medio del planteamiento de problemas más simples, es decir, de menor grado de dificultad, e) Resolver actividades de aprendizaje asignados por el profesor en su grupo de trabajo, que comprenden actividades de: exploración de contenidos previos, introducción de variables, síntesis y transferencia de contenidos a través de ejercicios, preguntas para la comprensión, problemas abiertos y más precisos; f) Puesta en común a través de presentaciones orales, póster y/o carteles: Una vez que ya todo está planeado, se lleva a cabo la investigación y se resuelven los pequeños problemas; los resultados son expuestos al grupo curso, donde se analizan las respuestas, el método de solución utilizado, dando la oportunidad de confrontar las dificultades encontradas; g) Contrastar y evaluar la resolución de los problemas más pequeños, que genera el cruce de información de varias investigaciones y generalizaciones realizadas y analizadas en la puesta en común. h) La solución de los problemas más acotados lleva a los estudiantes a integrar sus conocimientos para encontrar la solución al problema integrador.

El papel de los ejercicios en la propuesta de aprendizaje significativo, a través de la resolución problemas (ASARP), es el siguiente: a) Fomentar el uso de herramientas matemáticas necesarias para la interpretación, descripción y comprensión de los contenidos en Física; b) Evidenciar la aplicación y transferencia de contenidos, a través de la derivación e integración de parámetros de cinemática; c) Permitir que cuando los alumnos se enfrentan con los ejercicios puedan reconocer las condiciones físicas que son necesarias para la resolución de los mismos (condiciones físicas iniciales y finales); d) Eliminar la asociación, bastante generalizada, que hacen los alumnos de la Física, relacionando cada pregunta de un ejercicio con la fórmula para su resolución; e) A través de los ejercicios a resolver, se establece un método único de abordar la solución de todos los ejercicios de cinemática, de manera similar, por medio del cálculo diferencial.
Un aspecto muy interesante es analizar los nuevos interrogantes que surgen a partir de los resultados alcanzados en la búsqueda de la solución del problema original, haciendo ver cómo el trabajo sobre la solución de un problema raramente se cierra, sino que provoca nuevos interrogantes a partir de sus respuestas

\section{PROBLEMAS DE AULA}

A continuación, se muestra el problema integrador de contenido y la secuencia de problemas acotados que se presentan para trabajar en el aula y abordar los contenidos de cinemática, que son necesarios adquirir para resolver el problema.

\section{Problema Integrador: preparados para disfrutar de la Física y la emoción}

El Libro Guinness de Récords Mundiales cita al paracaidismo como el deporte, no mecánico, que provee la mayor velocidad a un individuo. La idea del paracaidismo no es nueva. Leonardo da Vinci pensó en el paracaidismo y dejó algunos dibujos que mostraban cómo podría funcionar un dispositivo como ese. En Francia, durante el siglo XVIII, Sébastien Lenormand, J. P. Blanchard y André Jaques Garnerin consideraron seriamente la caída desde las alturas e hicieron saltos experimentales desde globos de aire caliente. ¿Qué se experimenta al saltar desde un helicóptero y abrir el paracaídas? De acuerdo con esta introducción, responda a las siguientes preguntas:

1. ¿Por qué al lanzar hacia abajo o dejar caer un cuerpo desde una cierta altura su rapidez aumenta? y ¿Por qué al lanzar un objeto verticalmente hacia arriba, ésta disminuye?

2. ¿Qué tipos de movimiento puede adquirir un paracaidista?

3. a) ¿En qué tramos de la caída de un paracaidista la rapidez aumenta?

3. b) ¿Existe algún tramo en la caída de un paracaidista donde su rapidez disminuya?

3. c) ¿Existe algún tramo en caída del paracaidista donde su rapidez sea constante?

4. a) ¿Qué tipo de movimiento puede tener el helicóptero del problema enunciado? Explíquelo.

4. b) ¿Qué valor de aceleración puede adquirir el helicóptero? ¡Investigue valores reales!

4. c) Represente en un gráfico la variación de las coordenadas versus el tiempo del paracaidista (sin valores), desde el instante que se deja caer hasta llegar al piso en los casos: a) el helicóptero está suspendido en el aire; b) el helicóptero está en movimiento rectilíneo uniforme. 
5. a) ¿Qué puede decir de la aceleración de una bomba que se deja caer y de los proyectiles después de lanzados?

5. b) ¿Cuál es el valor de la aceleración, en los casos anteriores?

5. c) Encuentre la ecuación de movimiento que describa la caída del paracaidista para cualquier instante de tiempo hasta el momento en que se abra el paracaídas.

Problema $\mathbf{N}^{\mathbf{0}}$ 1. Las aplicaciones actuales del helicóptero son innumerables, abarcando misiones civiles y militares: transporte de pasajeros, ambulancia, fumigación, vigilancia, salvamento, etc. Los modelos utilizados se pueden ver en diferentes misiones y ámbitos. ¿Qué sucede cuando se salta de un helicóptero suspendido y luego se abre el paracaídas?

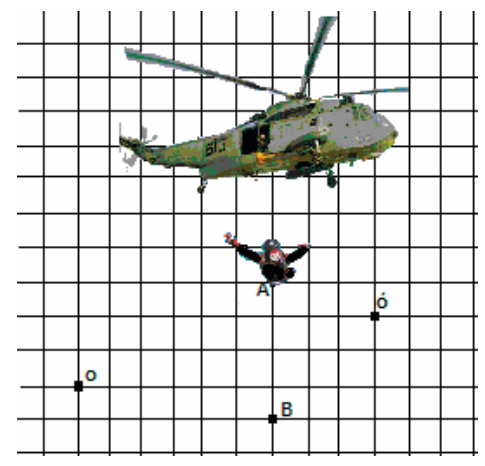

Figura 1. Caída de un paracaidista.

a) Describa el movimiento del paracaidista que se muestra en la figura 1, desde que se deja caer, se abre su paracaídas, hasta que llega al suelo.

b) Represente en un gráfico: a) la trayectoria del paracaidista, b) la rapidez y c) la aceleración que actúa sobre el paracaidista (sin valores).

c) Considere un sistema de referencia, en el punto $\mathrm{O}$, de la figura 1, e investigue acerca de los valores de altura apropiados para dejarse caer; asigne valores a cada cuadrado en la figura 1 y los instantes de tiempo que pasa por A y B. Encuentre: a) El vector posición del paracaidista al pasar por A y B; b) el desplazamiento entre A y B; c) la rapidez media; $d$ ) la velocidad media, d) repita las preguntas a, b, c y d, para el sistema de referencia en $\mathrm{O}^{\prime}$; d) Compare los resultado de: desplazamiento, rapidez media y velocidad media obtenidos en $\mathrm{O}$ y $\mathrm{O}$ '.

d) Asigne un valor a la rapidez del paracaidista al pasar por el punto $\mathrm{A}, \mathrm{y}$ encuentre las ecuaciones que describen su movimiento hasta llegar a B, donde se abre el paracaídas y cambia su rapidez.
Problema $\mathbf{N}^{\mathbf{0}}$ 2. Suponga que el helicóptero del caso anterior está en movimiento, entonces:

a) ¿Qué tipo de movimiento puede tener?

b) ¿Qué tipo de movimiento puede tener un paracaidista al saltar de él?

c) Represente en un gráfico el cambio de coordenada del helicóptero y el paracaidista.

Para cada tipo de movimiento que puedan adquirir el helicóptero y el paracaidista, asigne condiciones iniciales (valores iniciales de posición, rapidez y aceleración) y encuentre para cada caso las ecuaciones de movimiento que lo describen.

Problema $N^{0}$ 3. Durante la Segunda Guerra Mundial, millares de soldados se lanzaron desde aviones en paracaídas. Después de la guerra, muchos de estos veteranos continuaron lanzándose en paracaídas por diversión. ¿Qué sucede cuando se salta en paracaídas de un avión que se mueve con una rapidez $\mathrm{V}_{\mathrm{o}}$ en pleno vuelo y se abre el paracaídas? ¿Cómo se describe matemáticamente el movimiento del avión?; ¿Cómo se describe matemáticamente el movimiento del paracaidista? ¿Qué condiciones iniciales son necesarias conocer? Explique y fundamente su respuesta.

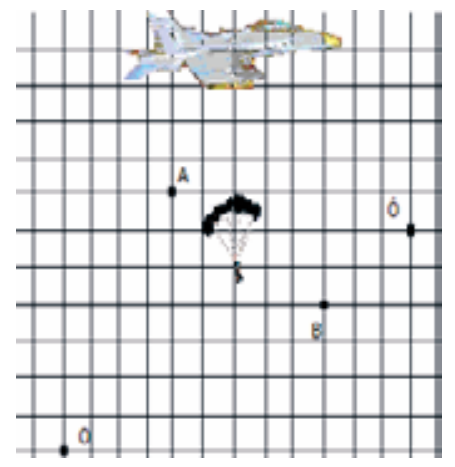

Figura 2. Caída de un paracaidista desde un avión.

a) Describa el movimiento completo del paracaidista.

b) Represente en un gráfico el cambio de coordenada del paracaidista y del avión simultáneamente.

c) Realice un gráfico que muestre cómo cambia la rapidez del paracaidista y del avión.

d) Considere un sistema de referencia, asigne valores a las cuadrículas, la rapidez inicial del avión y determine la posición, velocidad media y rapidez media, del avión y del paracaidista para los sistemas $\mathrm{O}$ y $\mathrm{O}^{\prime}$. Encuentre la ecuación que describe la caída del paracaidista hasta el momento de abrir su paracaídas. Para un observador en $\mathrm{O}$ (asigne valores iniciales de rapidez y posición del paracaidista). 
e) Asigne un valor a la posición, rapidez inicial y aceleración resultante en el instante que se abre el paracaídas y describa su movimiento para cualquier instante de tiempo en ese tramo.

\section{METODOLOGÍA DE LA INVESTIGACIÓN}

Se diseña y elabora una metodología para E-A por medio de problemas con sus correspondientes AA, a resolver en grupo y de forma individual en el aula, con base en un problema integrador y una serie de problemas más acotados que permiten abordar todos los contenidos de la unidad programática de cinemática, de la asignatura de Física I, que se cursa en las carreras de Ingeniería en Ejecución, en la Universidad del Bío-Bío (Chile).

Debido al uso indiscriminado de fórmula matemáticas en los libros de textos universitarios y en las clases de Física dictadas, actualmente, en la mayoría de las aulas universitarias en estos contenidos, se plantea, en este trabajo, usar las herramientas matemáticas conocidas del cálculo diferencial (derivación e integración) para obtener las expresiones matemáticas que describen el fenómeno físico en estudio.

Durante el transcurso del semestre, dos grupos de estudiantes (experimental y control) reciben una intervención metodológica de aula distinta, en el mismo horario y contenidos; estas son: a) Grupo Experimental, con base en problemas a resolver y uso de cálculo diferencial, con sus correspondientes AA, donde se aprende al investigar y compartir significados en el trabajo colaborativo; b) Grupo Control, que trabaja con metodología tradicional, clases expositivas en las que se entregan los contenidos en forma acabada, se realizan demostraciones que permiten encontrar las fórmulas generales para cada caso, y que posteriormente se utilizan para resolver ejercicios en la clase y en las prácticas.

Además del tiempo asignado por el programa (24 horas), se utilizaron cuatro horas adicionales para la aplicación de la prueba inicial y final respectivamente. Así, la secuencia total se extendió por un lapso de aproximadamente 1 mes, con una carga horaria de 8 horas semanales de teoría, práctica y laboratorio.

De acuerdo a la investigación, para comprobar cómo influyen las variables, mencionadas anteriormente, de la metodología propuesta (E-A), basada en problemas y actividades de aprendizaje, se utiliza un diseño de investigación CUASI-EXPERIMENTAL [24], con dos grupos, pre y post-test. Además, se realiza un análisis descriptivo y cualitativo de la información.
La investigación propone establecer la influencia de la propuesta en el aprendizaje de cinemática, para lo cual se plantean los siguientes interrogantes de investigación:

a) ¿Qué influencia ejerce la metodología empleada para enseñar y aprender en las estrategias de aprendizaje (calidad del aprendizaje) adquiridas por los estudiantes?

b) ¿Qué influencia ejerce la propuesta metodológica en el rendimiento académico de los estudiantes sometidos a la investigación?

c) ¿Qué percepción o valoración realizan los alumnos sometidos a la investigación acerca de la propuesta metodológica?

\section{Instrumentos de recolección de la información}

a) Las estrategias de aprendizaje se midieron a través del Inventario de R. Schmeck [25] que está formado por 55 enunciados distribuidos en: Procesamiento Elaborativo (PE); Procesamiento Metódico (PM); Procesamiento Profundo (PP); Retención de Hechos (RH). [26, 2].

b) Rendimiento académico: se mide a través de test y certamen de carácter formal, elaborado por un equipo de cuatro docentes que dictan la asignatura algunos con metodología tradicional y otros experimental, lo que asegura la validez del contenido; la confiabilidad del instrumentos de medidas se determina a través de la prueba de Kuder Richardson (KR20) para la fiabilidad, que considera ítems con respuesta correcta o incorrecta (escala dicotómica), resultado que se corresponde al coeficiente alpha de Cronbach [27]. Los alumnos se clasificaron, según su rendimiento académico, en dos modalidades, aprobado (60-100) y reprobado (0-59). La confiabilidad de los instrumentos de evaluación son: para el certamen de 0,91 muy buena, y la del test 0,72 buena.

c) Encuesta de Valoración: El objetivo del cuestionario es establecer la valoración que hacen los alumnos en la asignatura de Física a través de una encuesta abierta, con relación a la metodología para (E-A), actividades de aprendizaje y problemas presentados para trabajar en el aula, además de su grado de satisfacción y percepción acerca del aprendizaje adquirido durante el transcurso del semestre.

\section{MUESTRA}

La muestra que se utiliza para poner a prueba la hipótesis la constituyen dos cursos de Física I, con un total, aproximado, de 108 alumnos de la Universidad del BíoBío, Concepción, Chile. 


\section{RESULTADOS}

Estrategias de aprendizaje: En relación a la propuesta metodológica de aula y el procesamiento de la información, registrado a través del inventario de estrategias de aprendizaje aplicado en dos ocasiones, antes y después de aplicar la propuesta metodológica. Los datos obtenidos, en ambas mediciones, son analizados por categorías. Los cambios establecidos entre ambas mediciones se muestran en la tabla 1 , donde se puede apreciar que los alumnos sometidos a la propuesta metodológica logran cambios significativos en su forma de procesar la información, pasando de un modo superficial y reiterativo a uno más profundo y elaborativo, condición necesaria para lograr el aprendizaje significativo, lo que se corrobora por la prueba de Mc-Nemar que establece si existen cambios significativos entre las mediciones [28].

Tabla 1. Resultados estadísticos de las categorías del inventario en dos mediciones.

\begin{tabular}{|l|c|c|}
\hline F. Estrategias & $\left(\chi^{2}\right)$ & $(\mathbf{P})$ \\
\hline P. Elaborativo & 1,16 & 0,281 \\
\hline E. Metódico & 0,03 & 0,87 \\
\hline P. Profundo & 7,76 & 0,005 \\
\hline R. Hechos & 0,6 & 0,44 \\
\hline
\end{tabular}

$\chi^{2}=$ Estadístico; $\mathrm{P}=$ Nivel de significado

El gráfico 1 representa el porcentaje de respuestas en el eje horizontal versus las categorías del inventario de estrategias de aprendizaje en el eje vertical; expresadas a través de datos, en dicotómicos, estas categorías son: Procesamiento elaborativo bajo la normal (PEBN) y sobre la normal (PESN); Estudio metódico bajo la normal (EMBN) y sobre la normal (EMSN); Procesamiento profundo bajo la normal (PPBN) y sobre la normal (PPSN); y, finalmente, la Retención de hechos bajo la normal (RHBN) y sobre la normal (RHSN). Del análisis del gráfico se deduce que la categoría procesamiento profundo sobre y bajo la normal (PPSN y PPBN) muestra una mayor variación entre las dos mediciones, lo que se corrobora con la prueba de Mc-Nemar, que registra cambio significativo al $99 \%$ $(p=0,01)$. Los otros factores muestran variaciones pero éstas no son significativas al $95 \%(0,05)$.

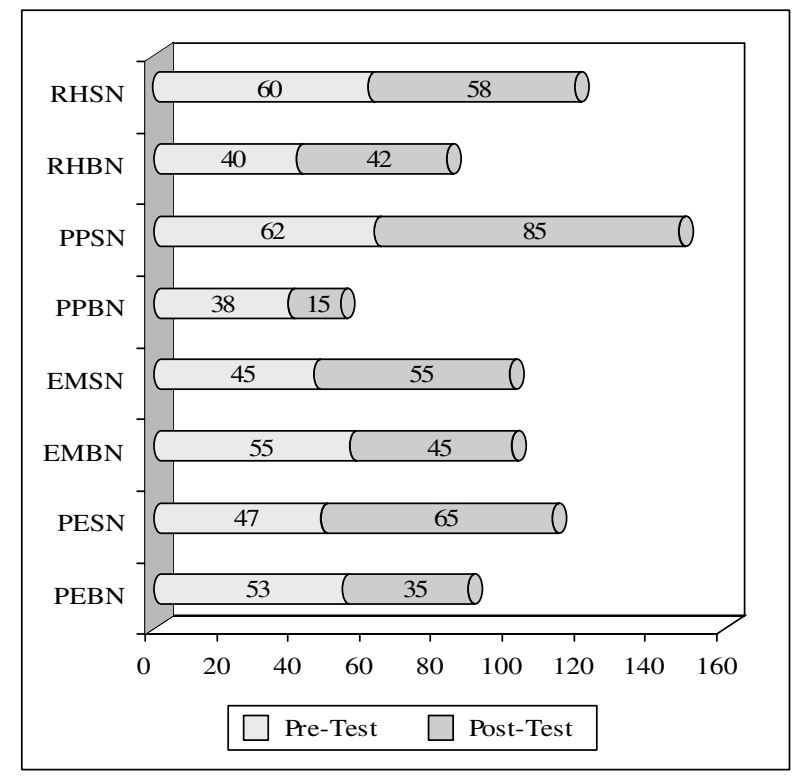

Gráfico 1. Porcentaje por respuestas versus las categorías del inventario de estrategias.

El gráfico 2 muestra los resultados alcanzados en las variables procesamiento profundo y elaborativo simultáneamente en dos mediciones, pre-test y pos/test, representadas en el eje horizontal, donde se ubican las variables: procesamiento profundo y elaborativo sobre y bajo la normal (PPESN y PPEBN); en el eje vertical, el porcentaje de respuestas que cumple la condición, es decir, la condición de aprendizaje significativo, implica alcanzar procesamiento profundo y procesamiento elaborativo sobre la normal al mismo tiempo.

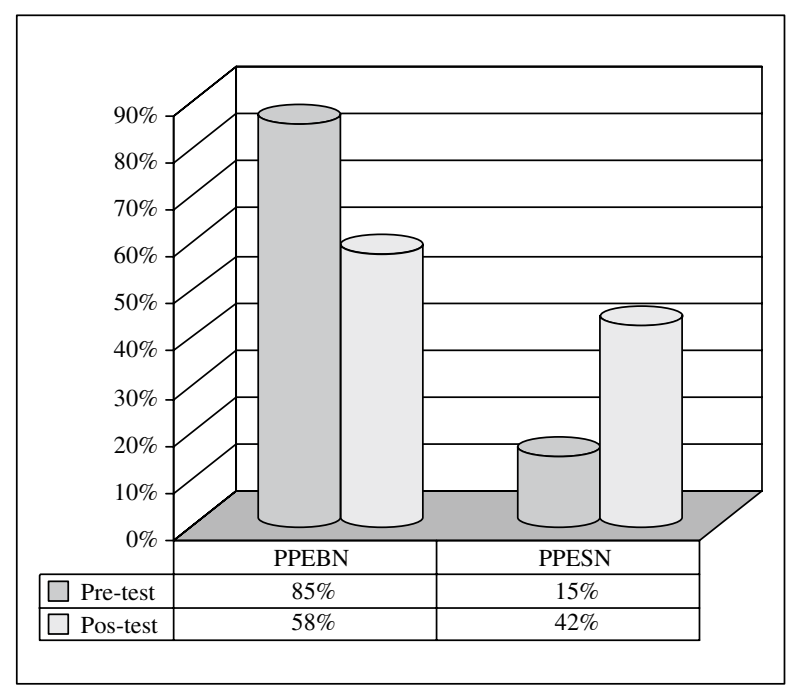

Gráfico 2. Porcentaje de alumnos con las condiciones para el aprendizaje significativo, antes y después de aplicar la propuesta metodológica. 
A partir del análisis del gráfico 2, se puede afirmar que la propuesta metodológica, con base en problemas en el aula, modifica la forma de procesar la información, pasando de un procesamiento superficial y reiterativo a uno profundo y elaborativo, lo que se corrobora estadísticamente por la prueba de Mc-Nemar, que establece un estadístico $\chi^{2}=9,48$ mayor al valor crítico, por lo tanto, se registra cambio significativo al 99,8\% ( $\mathrm{p}=0,002)$, es decir, después de aplicar la propuesta metodológica, una mayor cantidad de alumnos es capaz de relacionar, comprender, abstraer y transferir los contenidos aprendidos, condiciones necesarias para el aprendizaje significativo [29].

\section{RESULTADOS RENDIMIENTO ACADÉMICO}

El gráfico 3 muestra los resultados obtenidos en la aplicación del Test $\mathrm{N}^{\circ} 1$, que establece los conocimientos previos en los contenidos de vectores y parámetros de cinemática, en los grupos experimental y control en forma simultánea, antes de intervenir en el grupo experimental.

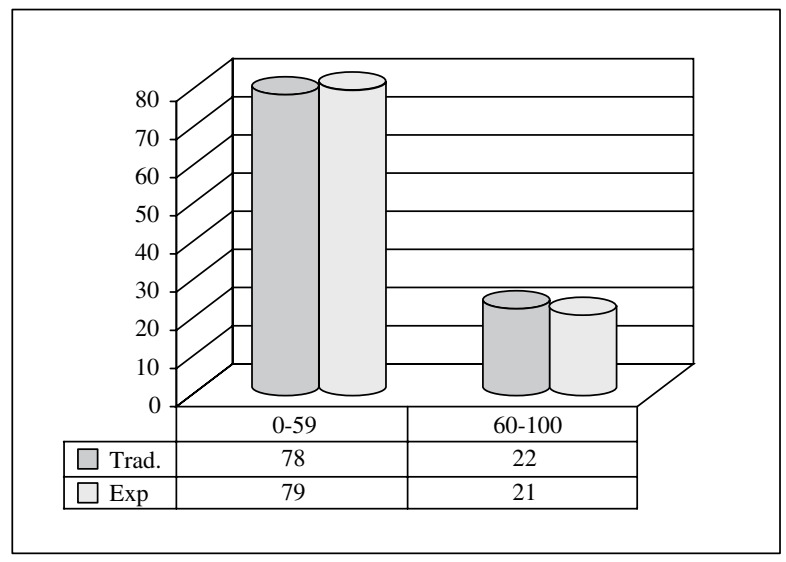

Gráfico 3. Rendimiento en una evaluación antes de aplicar la propuesta metodológica.

Del gráfico 3 se observa que los alumnos no presentan diferencias significativas en el rendimiento académico antes de aplicar la propuesta metodológica con base en problema ASARP, lo que se comprueba a través de las medias [28] con un 91\% ( $\mathrm{p}=0,8637)$, no existiendo diferencias significativas entre los grupos.

El gráfico 4 muestra los resultados alcanzados en los grupos experimental y control, al ser enfrentados a un mismo instrumento de evaluación (certamen $\mathrm{N}^{\mathrm{o}} 1$, formal), después de realizar las correspondientes intervenciones, propuesta metodológica con base en resolución de problemas para el aprendizaje significativo (ASARP) en el grupo experimental y la propuesta tradicional en el grupo control.

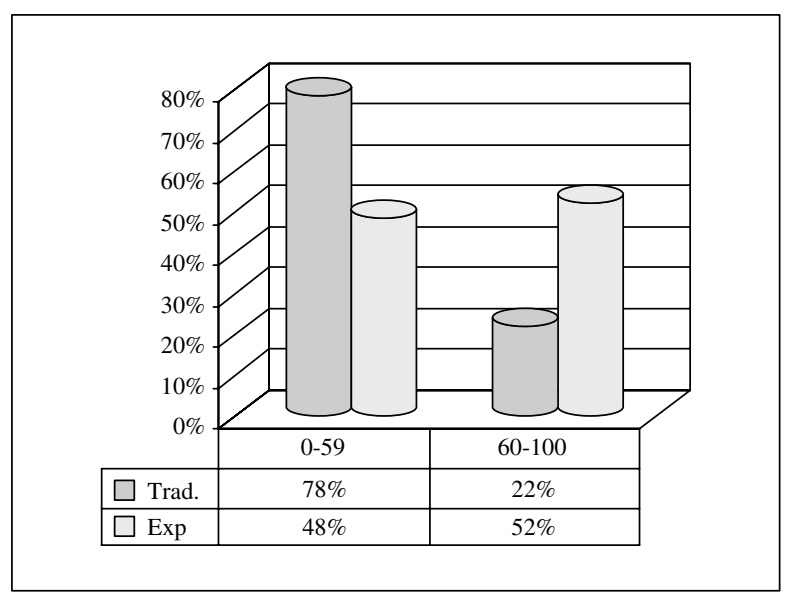

Gráfico 4. Influencia de la propuesta metodológica en el rendimiento (certamen).

El eje horizontal del gráfico 4 recoge el porcentaje de alumnos que alcanza un determinado rendimiento académico, y en el eje vertical, la propuesta metodológica de aula, tradicional o con base a problemas (ASARP). Aquí se observa que los alumnos del grupo experimental obtuvieron un mejor rendimiento académico, que los alumnos del grupo control, diferencia que es significativa a $99,97 \%(\mathrm{p}=0,0003)$, según la prueba de las media con un estadístico de $\chi^{2}=12,84$ mayor que el valor crítico, es decir, se corrobora la hipótesis acerca de la influencia de la metodología basada en ASARP en el rendimiento académico con un $99,97 \%$.

En el gráfico 5 se presentan los resultados alcanzados en el rendimiento académico por el grupo experimental y control (en porcentaje de alumnos), sólo en los contenidos del problema de cinemática en una y dos dimensiones (a diferencia del gráfico 4 que considera una mayor cantidad de contenido, circular, movimiento relativo y newtoniano), del instrumento de evaluación (certamen $\mathrm{N}^{\mathrm{o}} 1$, formal), después de aplicar metodología basada en resolución de problemas para el aprendizaje significativo (ASARP) en el grupo experimental. 


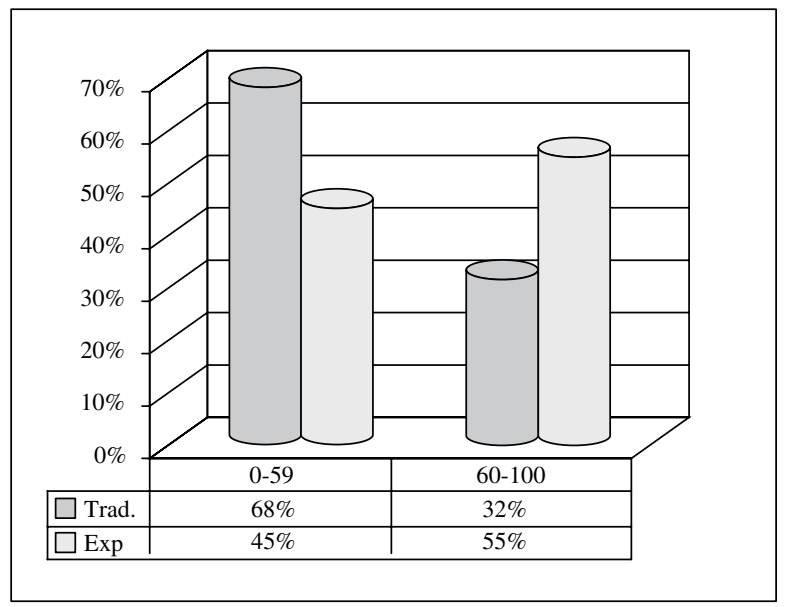

Grafico 5. Influencia de la propuesta metodológica en el rendimiento académico.

Del gráfico 5 se establece que los alumnos que fueron enfrentados a la resolución de problemas obtienen un mejor rendimiento académico en los contenidos de cinemática que los alumnos que trabajan con metodología tradicional. Lo que se corrobora a través de la prueba estadística Ji-cuadrado, que establece diferencias significativas a $99,9998 \%$ ( $p=0,0002$ y valor estadístico $\chi^{2}=13,76$ ) a favor del grupo experimental. Luego se confirma la hipótesis acerca de la influencia de la metodología basada en ASARP en el rendimiento académico.

\section{VALORACIÓN PROPUESTA METODOLÓGICA}

El gráfico 6 muestra los resultados obtenidos de la encuesta de valoración abierta, agrupados en tres categorías: buena, omite y mala. En él se observa la valoración hecha por los estudiantes acerca de la propuesta metodológica y las actividades de aprendizaje usada en el aula para promover la adquisición de aprendizaje significativo.

\section{ENCUESTA VALORACIÓN}

P.1 ¿Qué opina de la forma de trabajar en el aula en este curso?

P.2 ¿Cuál es su grado de satisfacción (1-59= Mala; $60-100=$ Buena) hasta el momento en este curso?

P.3 ¿Qué opina acerca de los problemas y actividades de aprendizaje utilizados en este curso?

P.4 ¿Qué opina acerca del trabajo en equipo y la interacción en el aula al trabajar de esta forma?

P.5 ¿Qué opina acerca de tu nivel de comprensión alcanzado en la mayor parte de los contenidos trabajados en el aula a lo largo del curso?
P.6 ¿Qué opina acerca de la forma de abordar los ejercicios de cinemática, para obtener ecuaciones que describen los movimientos? (cálculo diferencial)

P.7 ¿Qué opina acerca de las exposiciones que se realizan por los alumnos en las puestas en común en el aula con esta forma de trabajo?

P.8 ¿Qué opina acerca de esta forma de trabajar en el aula y la capacidad de relacionar los temas con tu entorno?

P.9 ¿Qué opina acerca de esta forma de trabajar en el aula y la capacidad para sustentar, defender y respetar ideas propias y de tus compañeros?

P.10 ¿Qué opina acerca de la forma de trabajo en el aula y el uso de las habilidades para buscar, resumir, organizar, transferir y comunicar información?

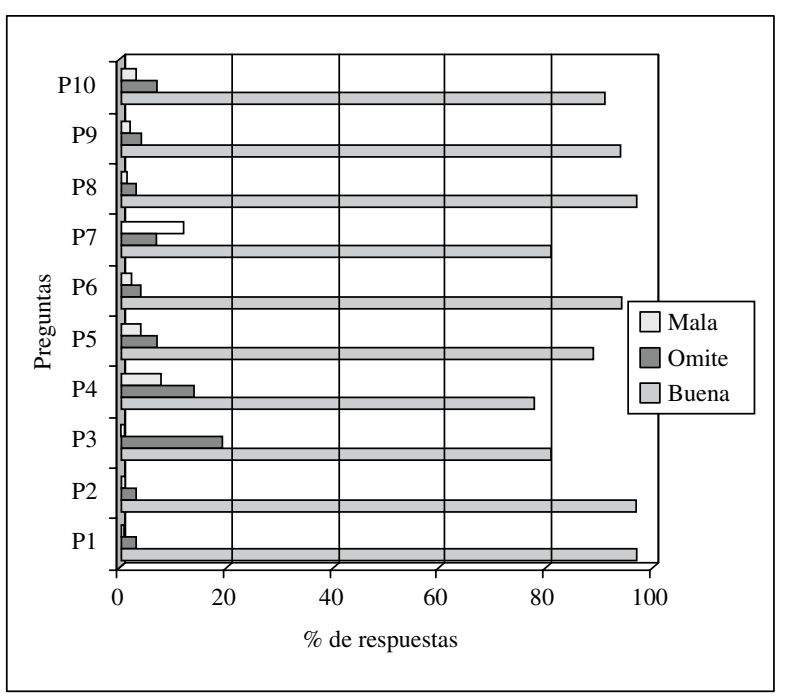

Gráfico 6. Valoración de la propuesta metodológica.

De los resultados de la encuesta se deduce que la ventaja de trabajar en equipo y en este marco colaborativo proviene: de la interacción social, del trabajo en grupo de 5 alumnos, del valor cognitivo que tiene para cada alumno explicar problemas o que se lo expliquen y de las actividades de aprendizaje empleadas, que implican uso de cálculo diferencial y que son común para todos los grupos, lo que promueve la competición entre los grupos (a resolver antes los problemas), manteniendo su atención centrada en la tarea académica [1].

\section{CONCLUSIONES}

A partir de los resultados obtenidos en esta investigación, se aportan fundamentos que permiten presentar las siguientes conclusiones, que pueden ser de relevancia para futuras investigaciones en el tema. 
Constituye una alternativa válida para mejorar la calidad del aprendizaje en los alumnos, lo que se infiere al establecer la influencia de la propuesta metodológica de aula en las estrategias de aprendizaje, que presentan cambios significativos en una categorías del cuestionario sobre el procesamiento profundo de la información $99,5 \%(\mathrm{p}=0.005)$, que se relaciona con la transferencia y abstracción de contenidos. Por otra parte, del Gráfico $\mathrm{N}^{\circ} 2$, se puede afirmar que también se modifica la forma de procesar la información, pasando de un procesamiento superficial y reiterativo a uno profundo y elaborativo, lo que se avala, estadísticamente, por la prueba de Mc-Nemar que registra cambio significativo al 99,8\% ( $\mathrm{p}=0,002)$, es decir, después de aplicar la propuesta metodológica, una mayor cantidad de alumnos es capaz de relacionar, comprender, abstraer y transferir los contenidos aprendidos, condición requerida para el aprendizaje significativo [29], que implica alcanzar procesamiento profundo y elaborativo sobre la normal al mismo tiempo.

La aplicación de aprendizaje con base en problemas ASARP, como metodología para enseñar y aprender, en cinemática, permite hacer algunas afirmaciones con relación a la propuesta metodológica y el rendimiento académico.

I. Los alumnos del grupo experimental obtuvieron un rendimiento académico, significativamente mayor en la evaluación de los contenidos de cinemática en una y dos dimensiones en la primera evaluación formal (certamen $\mathrm{N}^{\mathrm{o}}$ 1) que los alumnos del grupo control, que trabajaron con metodología tradicional. Esto se comprueba a través de la prueba estadística de la media, que muestra (estadístico $\chi^{2}=13,76$ ) cambio significativo al 99.9998 ( $\mathrm{p}=0,0002)$. Por otra parte, al comparar el rendimiento en el certamen $\mathrm{N}^{\mathrm{o}}$ 1, incluyendo todos los contenidos, se confirma lo anterior con (estadístico $\chi^{2}=12,84$ ) cambio significativo al 99,9997\% ( $\mathrm{p}=0,0003)$ a favor del grupo experimental.

II. Los alumnos del grupo experimental muestran cambios significativos en la adquisición de los contenidos de cinemática, lo que se refleja en los resultados obtenidos entre la primera y segunda medición test y certamen, que muestra cambio significativo al 99,9999\% ( $\mathrm{p}=0,000)$ según la prueba de McNemar, (estadístico $\chi^{2}=24,74$ ), lo que permite inferir que el uso de problemas y actividades de aprendizaje trabajadas en forma colaborativa en el aula y fuera de ella incide significativamente en el rendimiento académico. Por otra parte, de los resultados se deduce que los estudiantes aplicaron y posiblemente transfirieron los contenidos a situaciones nuevas distintas a las trabajadas en los problema de la clase, que se refleja en mejores calificaciones del certamen $\mathrm{N}^{\mathrm{o}}$ 1, que incluye situaciones distintas a las trabajadas en el aula, nuevas, lo que indicaría que su aprendizaje fue significativo.

En relación a la metodología utilizada en la asignatura, para enseñar y aprender, se afirma que se elimina la frontera entre práctica y teoría, lo que se logra a través de la resolución de problemas en el aula y la investigación de los contenidos, de situaciones planteadas en todo momento, lo que motiva la interacción entre los estudiantes, entre los estudiantes y el material educativo, entre los estudiantes y el profesor. Así, se promueve la negociación de significados y la transferencia de contenidos, condiciones del aprendizaje significativo.

De los resultados alcanzados en la encuesta de opinión al grupo experimental se puede inferir que la propuesta metodológica, con base en problemas, promueve en el estudiante las habilidades de buscar, resumir, organizar, transferir y comunicar información. Por otra parte, en relación al trabajo en equipo, se puede afirmar que se desarrolla la capacidad para sustentar, defender y respetar ideas propias y de los compañeros, aprendiendo mucho más de manera activa y participativa. Así, se motiva la interacción social entre la tríada alumno, profesor y materiales educativos (problemas) para compartir y negociar significados, lo que se corrobora con el alto grado de satisfacción de los alumnos con respecto al aprendizaje adquirido, que opinan favorablemente y reconocen las ventajas y cualidades de la propuesta, que mejora la interacción social en el aula, es activa y participativa, promueve la búsqueda de información y favorece la construcción del conocimiento.

El uso del cálculo diferencial para describir matemáticamente un fenómeno y obtener las ecuaciones de movimiento (a través de la derivación e integración) elimina el uso reiterativo de fórmulas que promueven un aprendizaje superficial y reiterativo, lo que favorece el uso de las condiciones físicas iniciales y finales en la solución de los ejercicios [30]. Además, se promueve el aprendizaje significativo de los conceptos físicos involucrados en cada pregunta de un ejercicio, favoreciendo la interpretación física y no el uso indiscriminado de fórmulas, activando los principios facilitadores de aprendizaje, la diferenciación progresiva y la reconciliación integradora de los contenidos de cinemática, al eliminar el uso de ecuaciones para movimientos horizontales y verticales, en dos dimensiones y ser trabajados todos de forma similar. De este modo, se evita la fragmentación de los contenidos, la división de 
capítulos en subcapítulos, puesto que son trabajados todos como un gran bloque temático, lo que puede facilitar el aprendizaje significativo.

En la aplicación del aprendizaje con base en problemas como metodología para enseñar y aprender, se observan dificultades para captar aprendizaje significativo que en su mayoría se corresponden con las enunciadas por [31], que son: a) Los alumnos están acostumbrados a escuchar pasivamente al profesor y memorizar, por tanto, cualquier cambio en la metodología de trabajo, provoca al principio inseguridad en los estudiantes; b) Los estudiantes no establecen relaciones entre los contenidos trabajados en las clases teóricas y las prácticas; c) El alumno no reconoce su carencia de conocimientos; d) Dificultades de representación e interpretación de los tipos de movimientos; e) Dificultades para diferenciar cantidades físicas que lleven a describir y explicar el movimiento; f) Dificultades para interpretar y describir movimientos a partir de sistemas de referencia no acostumbrados a usar por ellos; g) La comunicación de información, en general, es muy superficial.

\section{AGRADECIMIENTOS}

El soporte conceptual de este trabajo es aportado por el Proyecto $N^{\circ} 1071050$, financiado por el Fondo de Desarrollo Científico y Tecnológico de Chile (FONDECYT) y del proyecto interno de investigación N. ${ }^{\circ}$ 0611073/R de la Universidad del Bío-Bío.

\section{REFERENCIAS}

[1] I. Sánchez y P. Flores. "Influencia de una metodología activa en el proceso de enseñar y aprender Física". Journal of Science Education. Vol. $5 \mathrm{~N}^{\circ} 2$, pp. 77-83. Bogotá. Colombia. Julio 2004.

[2] I. Sánchez. "Validación de una metodología basada en actividades de aprendizaje con técnicas creativas para estudiantes universitarios". Journal of Science Education. Año 2. Vol. 2, pp. 86-90. Bogotá. Colombia. Julio 2001.

[3] A. Medina y M. Salvador. "Didáctica General". Prentice Hall. Madrid. España, pp. 103-127. 2002.

[4] J. Jorba y E. Casella. "La regulación y la autorregulación de los aprendizajes". Sintesis S.A. Vol. 1, pp. 124-234. Barcelona, España. 1997.
[5] F. Perales. "Didáctica de las Ciencias Experimentales”. Marfil S.A., pp. 209-306. España. 2000.

[6] D. Gil y J. Martínez. "A model for problem solving in accordance with scientific methodology". European Journal of Science Education. Vol. 5 No 4, pp. 447-455. Marzo 1983.

[7] D. Gil y J. Martínez. "La resolución de problemas de física". Vincens Vives-MEC, pp. 13-37. Madrid. España. 1987.

[8] D. Gil, C. Furió, P. Valdés, J. Salinas, J. Martínez, J. Guisasola, J. González, A. Dumas, M. Goffard y A. Pessoa. “ ¿Tiene sentido seguir distinguiendo entre aprendizaje de conceptos, resolución de problemas de lápiz y papel y realización de prácticas de laboratorio?". Enseñanza de las Ciencias. Vol. 17 $\mathrm{N}^{\mathrm{o}}$ 2, pp. 311-320. Junio 1999.

[9] D. Ausubel, J. Novak y H. Hanesian. "Psicología Educativa". Trillas. México. 1978.

[10] D. Ausubel. "Adquisición y retención del conocimiento. Una perspectiva cognitiva”. Paidós. Barcelona, España. 2000.

[11] M.A. Moreira. "Aprendizaje significativo: teoría y práctica”. Visor, pp. 3-100. Madrid, España. 2000 .

[12] J. Novak y D. Gowin. "Aprendiendo a aprender". Martínez Roca, pp. 19-76. Barcelona, España. 1988.

[13] L. Vygotsky. "Psicología y pedagogía". Akal, pp. 90-156. Madrid, España. 1979.

[14] I. Sánchez y F. Ramis. "Aprendizaje Significativo basado en Problemas". Revista Horizontes Educacionales. Vol. 1 Nº, pp. 101-111. Chillán. Chile. Noviembre 2004.

[15] T. Shuell. "Phases of meaningful learning". Review of Educational Research. Vol. 60, pp. 531-547. 1990.

[16] D. Ausubel, J. Novak and H. Hanesian. "Psicología Educativa”. Trillas, pp. 42-500. México. 1997.

[17] L Ribeiro and M. Mizukami. "Problem-based learning: a student evaluation of an implementation in postgraduate engineering education". European 
Journal of Engineering Education. Vol. $30 \mathrm{~N}^{\mathrm{o}} 1$, pp. 137-149. 2005.

[18] I. Sánchez, M.A. Moreira y C. Caballero. "Aprendizaje significativo de la cinemática a través de resolución de problemas y uso de cálculo diferencial en estudiantes universitarios". Enseñanza de las Ciencias. Número Extra, pp. 210-220. Barcelona, España. 2005

[19] B. Duch. "Problem-based Learning in Large and Very Large Classes". En: Duch, B.J., S. E. Groh y D. E. Allen (Eds), The Power of Problem-based Learning: A Practical 'How to' for Teaching Courses in any Discipline, Sterling, VA: Stylus, pp. 149-163. 2001.

[20] D. Allen y B. Duch. "Thinking towards solutions: problem-based learning activities for general biology". Philadelphia: Saunders College Publishing. 1998.

[21] B. Duch. "Problems: A Key Factor in PBL". En: About Teaching 50. Spring, p. 78. 1996.

[22] B. Duch. "A Bad Day for Sandy Dayton the Physics of Accident Reconstruction". J. College Science Teaching. Vol. $30 \mathrm{~N}^{\mathrm{o}}$ 1, pp. 17-21. 2000.

[23] N. Schultz y H. Christensen. "Seven-step problembased learning in an interaction design course European". Journal of Engineering Education. Vol. $29 \mathrm{~N}^{\mathrm{O}}$ 4, pp. 533-541. 2004.

[24] L. Cohen y L. Manion. "Métodos de investigación educativa”. La muralla S.A., pp. 10-100. España. 1990.

[25] I. Truffello y F. Pérez. Adaptación en Chile del "Inventory of Learning Processes de R. Schmeck". Boletín de Investigación. Pontificia Universidad Católica de Chile. Vol. 15, pp. 109-120. Diciembre 1998.

[26] H. Alvarado, I. Sánchez y M. Uribe. "Relación entre estrategias de aprendizaje y rendimiento académico en estudiantes Universitarios". Boletín de Investigación. Pontificia Universidad Católica de Chile. Vol. 15, pp. 70-88. Diciembre 2000.

[27] E. López. E. Barajas y R. Pérez. "Pedagogía Experimental I". Tomo II, pp. 297-412. UNED. Madrid, España. 1997.
[28] R. Sierra. "Técnicas de investigación Social". Teoría y ejercicios. Paraninfo S.A., pp. 550-700. España. 2003.

[29] I. Sánchez. "Aprendizaje Significativo y resolución de problemas, simposio trilogía para el aprendizaje significativo en ciencia”. Acta del II congreso Internacional de Estilos de Aprendizaje. Universidad de Concepción. Concepción, Chile. Enero 2006.

[30] J. Perales. "Resolución de problemas". Editorial Síntesis, S.A., pp. 3-25. Madrid, España. 2000.

[31] A. Oñorbe y J Sánchez. "Dificultades en la enseñanza-aprendizaje de los problemas de Física y Química I. Opiniones de los alumnos". Enseñanza de las Ciencias. Vol. 14 NN$^{\circ}$ 2, pp. 165-170. Barcelona, España. Junio 1996.

\section{ANEXO: ACTIVIDADES DE APRENDIZAJE}

Las ecuaciones generales de los movimientos en estudio que permiten determinar la velocidad, posición y aceleración en cualquier instante de tiempo se encuentran a través del uso del cálculo diferencial. Es decir, con la integración o derivada cuando corresponda

$$
\begin{aligned}
& \vec{a}(t)=\frac{d \vec{v}(t)}{d t} \Leftrightarrow \vec{v}(t)=\int \vec{a}(t) \bullet d t \\
& \vec{v}(t)=\frac{d \vec{r}(t)}{d t} \Leftrightarrow \vec{r}(t)=\int \vec{v}(t) \bullet d t
\end{aligned}
$$

Actividad de Aprendizaje $\mathbf{N}^{\mathbf{0}}$ 1. Un niño lanza una pelota desde una altura inicial $(\mathrm{t}=0)$ de $10[\mathrm{~m}]$ hacia un amigo parado en el suelo, el cual la alcanza cuando pasa a $1[\mathrm{~m}]$ de altura. Si para $\mathrm{t}=1[\mathrm{~s}]$ la velocidad de la pelota es $\vec{v}(1)=16 \hat{i}+12 \hat{j}[\mathrm{~m} / \mathrm{s}]$. Responda las siguientes preguntas.

a) Haga un dibujo esquemático de la situación, fije un sistema de referencia

b) Determine las ecuaciones de movimiento, la posición y velocidad de la pelota.

c) ¿Qué distancia horizontal recorre la pelota hasta ser atrapada por el amigo?

Nota: En este ejercicio use $\mathrm{g}=10\left[\mathrm{~m} / \mathrm{s}^{2}\right]$,

para $\mathrm{t}=1[\mathrm{~s}], \overrightarrow{\mathrm{v}}_{1}(1)=16 \hat{\mathrm{i}}+12 \hat{\mathrm{j}}[\mathrm{m} / \mathrm{s}] ; \overrightarrow{\mathrm{a}}(\mathrm{t})=-10 \hat{\mathrm{j}}\left[\mathrm{m} / \mathrm{s}^{2}\right]$ 


\section{Condiciones iniciales}

Ecuaciones

$$
\begin{aligned}
& \overrightarrow{\mathrm{v}}(t)=\int-10 \hat{\mathrm{j} d t}[\mathrm{~m} / \mathrm{s}] \Rightarrow \overrightarrow{\mathrm{v}}(t)=(-10 \hat{\mathrm{j}} \bullet \mathrm{t}+\mathrm{c})[\mathrm{m} / \mathrm{s}] \Rightarrow \\
& (16 \hat{i}+12 \hat{\mathrm{j}})[\mathrm{m} / \mathrm{s}]=(-10 \hat{\mathrm{j}} \bullet 1+\mathrm{c})[\mathrm{m} / \mathrm{s}] \\
& 16 \hat{i}+22 \hat{\mathrm{j}}=c \Rightarrow \\
& \therefore \overrightarrow{\mathrm{v}}(t)=16 \hat{i}+(22-10 \mathrm{t}) \hat{\mathrm{j}}[\mathrm{m} / \mathrm{s}]
\end{aligned}
$$

Condiciones iniciales

$$
\begin{aligned}
& \mathrm{t}=0[\mathrm{~s}] ; \overrightarrow{\mathrm{r}}(0)=10 \mathrm{j}[\mathrm{m}] ; \overrightarrow{\mathrm{a}}(\mathrm{t})=-10 \hat{\mathrm{j}}\left[\mathrm{m} / \mathrm{s}^{2}\right] \\
& \overrightarrow{\mathrm{r}}(t)=\int(16 \hat{i}+(22-10 t) \hat{\mathrm{j}}) \mathrm{dt}[\mathrm{m}] \Rightarrow+ \\
& \overrightarrow{\mathrm{r}}(t)=\left(16 \hat{i}+\left(22 t-5 t^{2}\right) \hat{\mathrm{j}}+\mathrm{c}\right)[m] \Rightarrow 10 j=c \Rightarrow \\
& \therefore \quad \overrightarrow{\mathrm{r}}(t)=\left(16 \hat{i}+\left(10+22 t-5 t^{2}\right) \hat{\mathrm{j}}\right)[m]
\end{aligned}
$$

Condición de frontera final; $\overrightarrow{\mathrm{r}}_{\mathrm{y}}(t)=1 \hat{j}[m]$

$$
\begin{aligned}
& \left.1 \widehat{j}[m]=\left(10+22 t-5 t^{2}\right) \hat{j}[m]\right] \Rightarrow 1=10+22 t-5 t^{2} \\
& \Rightarrow 5 t^{2}-22 t-9=0 \Rightarrow t_{1}=-n o \exists \quad y \quad t_{2} \approx 4,8[s] \\
& \therefore \quad \overrightarrow{\mathrm{r}}_{\mathrm{x}}(t)=(16 \bullet 4,8 \hat{i})[m] \approx 77 \hat{i}[\mathrm{~m}]
\end{aligned}
$$

Actividad de Aprendizaje $\mathbf{N}^{\circ}$ 2. La figura muestra (para el instante $\mathrm{t}=1$ ) a un automóvil cuya ecuación de su velocidad es $\vec{v}_{A}(t)=(0,8 t+4) \hat{i}[\mathrm{~m} / \mathrm{s}]$ y un camión que para ese instante se mueve con una rapidez de $30[\mathrm{~m} / \mathrm{s}]$ y desacelera a razón constante de $2\left[\mathrm{~m} / \mathrm{s}^{2}\right]$ Entonces: encuentre las ecuaciones de movimiento del auto y el camión, la posición y velocidad donde se cruzan.

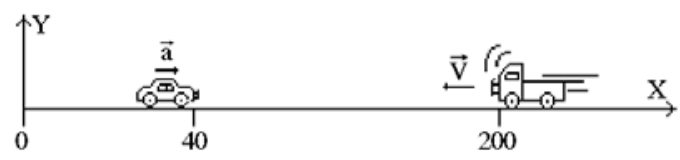

Condiciones Iniciales Auto.

$$
t=1[s] \Rightarrow \vec{r}(1)=40 \hat{i}[m] ; \overrightarrow{\mathrm{V}}_{\mathrm{A}}(\mathrm{t})=(0,8 \mathrm{t}+4) \hat{\mathrm{i}}
$$

La posición para todo instante de tiempo es:

$$
\begin{aligned}
& \overrightarrow{\mathrm{r}}_{\mathrm{A}}(\mathrm{t})=\int \overrightarrow{\mathrm{v}}_{\mathrm{A}}(\mathrm{t}) \bullet \mathrm{dt} \Rightarrow \overrightarrow{\mathrm{r}}_{\mathrm{A}}(\mathrm{t})=\int(0,8 \mathrm{t}+4) \hat{\mathrm{i}} d t[\mathrm{~m}] \\
& \overrightarrow{\mathrm{r}}_{\mathrm{A}}(\mathrm{t})=\left(0,4 \mathrm{t}^{2}+4 \mathrm{t}\right) \hat{\mathrm{i}}+\mathrm{c}[\mathrm{m}] \Rightarrow t=1[\mathrm{~s}] \Rightarrow \vec{r}(1)=40 \hat{i}[\mathrm{~m}] \\
& 40 \hat{i}=0,4 \hat{\mathrm{i}}+4 \hat{\mathrm{i}}+\mathrm{c} \Rightarrow 35,6 \hat{\mathrm{i}}=\mathrm{c} \\
& \therefore \overrightarrow{\mathrm{r}}_{\mathrm{A}}(\mathrm{t})=\left(0,4 \mathrm{t}^{2}+4 \mathrm{t}+35,6\right) \hat{\mathrm{i}}[\mathrm{m}] \\
& \overrightarrow{\mathrm{a}}(\mathrm{t})=\frac{\mathrm{d} \overrightarrow{\mathrm{v}}_{\mathrm{A}}(\mathrm{t})}{\mathrm{dt}}=\frac{d(0,8 \mathrm{t}+4) \hat{\mathrm{i}}}{d t}=0,8 \hat{i}\left[\mathrm{~m} / \mathrm{s}^{2}\right] \\
& \overrightarrow{\mathrm{a}}(\mathrm{t})=0,8 \hat{i}\left[\mathrm{~m} / \mathrm{s}^{2}\right]
\end{aligned}
$$

Condiciones iniciales para el camión

$$
\begin{aligned}
& \mathrm{t}=1[\mathrm{~s}] ; \quad \overrightarrow{\mathrm{r}}(1)=200 \hat{\mathrm{i}}[\mathrm{m}] ; \quad \overrightarrow{\mathrm{V}}_{\mathrm{C}}(1)=-30 \hat{\mathrm{i}}[\mathrm{m} / \mathrm{s}] ; \quad \vec{a}(t)=2 \hat{i}\left[\mathrm{~m} / \mathrm{s}^{2}\right] \\
& \vec{v}(t)=\int \vec{a}(t) d t \Rightarrow \vec{v}(t)=\int 2 \hat{i} d t[\mathrm{~m} / \mathrm{s}] \\
& \vec{v}(t)=(2 \hat{i}+c)[\mathrm{m}] \Rightarrow \mathrm{t}=1[\mathrm{~s}] \Rightarrow \overrightarrow{\mathrm{V}}_{\mathrm{C}}(1)=-30 \hat{\mathrm{i}}[\mathrm{m} / \mathrm{s}] \\
& \Rightarrow-30 \hat{\mathrm{i}}[\mathrm{m} / \mathrm{s}]=(2 t \hat{i}+c) \Rightarrow c=-32 \hat{i}[\mathrm{~m} / \mathrm{s}] \\
& \therefore \vec{v}(t)=(2 t-32) \hat{i}[\mathrm{~m}]
\end{aligned}
$$

La posición para todo instante de tiempo está dada por:

$$
\begin{aligned}
& \overrightarrow{\vec{r}}(t)=\int \vec{v}(t) d t \Rightarrow \overrightarrow{\vec{r}}(t)=\int(2 t-32) \hat{i} d t[m] \\
& \overrightarrow{\vec{r}}(t)=\left(t^{2}-32 t\right) \hat{i}+c \Rightarrow 200 \hat{i}[m]=-31[m] \widehat{i}+c \Rightarrow c=231 \hat{i}[\mathrm{~m}] \\
& \overrightarrow{\vec{r}}(t)=\left(t^{2}-32 t+231\right) \hat{i}[\mathrm{~m}]
\end{aligned}
$$

El instante en que se encuentra o cruzan:

$$
\begin{aligned}
& \overrightarrow{\mathrm{r}}_{\mathrm{A}}(\mathrm{t})=\overrightarrow{\vec{r}}(t) \\
& \left(0,4 \mathrm{t}^{2}+4 \mathrm{t}+35,6\right) \hat{\mathrm{i}}[\mathrm{m}]=\left(t^{2}-32 t+231\right) \hat{i}[m] \\
& 0,6 \mathrm{t}^{2}-36 t+195,4=0 \Rightarrow \mathrm{t}^{2}-100 t+326=0 \\
& t=3,38[\mathrm{~s}] \wedge t=96,6[\mathrm{~s}]
\end{aligned}
$$

Condición final; $\vec{r}_{A}(t)=\bar{r}_{C}(t)$

Se resuelve ecuación segundo grado y los valores positivos del tiempo se reemplazan en ecuaciones de posición y velocidad.

Una esquiadora se desliza sobre una rampa de lanzamiento, en el instante en que abandona la rampa lo hace con una rapidez de $90[\mathrm{~km} / \mathrm{h}]$ y un ángulo de $30^{\circ}$ respecto de la horizontal.

a) Encontrar las ecuaciones de movimiento. 
b) ¿Cuál es la altura máxima a la que se eleva la esquiadora.

c) ¿Cuál es su alcance, cuando alcanza una planicie a $5[\mathrm{~m}]$ del piso?

d) ¿Qué velocidad lleva al alcanzar la planicie?

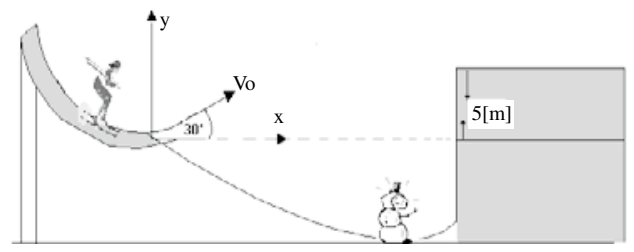

\section{Condiciones iniciales}

$\mathrm{t}=0[\mathrm{~s}]$

$\overrightarrow{\mathrm{r}}(0)=0 \hat{\mathrm{i}}+0 \hat{\mathrm{j}}[\mathrm{m}]$

$\overrightarrow{\mathrm{V}}_{\mathrm{C}}(1)=25 \cos 30 \hat{\mathrm{i}}+25 \operatorname{sen} 30 \hat{\mathrm{j}}[\mathrm{m} / \mathrm{s}]$

Ecuaciones

$\vec{a}(t)=-9,8 \hat{j}\left[m / s^{2}\right]$

$\vec{v}(t)=\int \vec{a}(t) d t \Rightarrow \vec{v}(t)=\int-9,8 \hat{j} d t[m / s]$

$\vec{v}(t)=(-9,8 \hat{j} t+c)[m] \Rightarrow \mathrm{t}=0[\mathrm{~s}]$

$\overrightarrow{\mathrm{V}}_{\mathrm{C}}(0)=20 \hat{\mathrm{i}}+12,5 \hat{\mathrm{j}}[\mathrm{m} / \mathrm{s}]$

$\Rightarrow 20 \hat{\mathrm{i}}+12,5 \hat{\mathrm{j}}=c \Rightarrow$

Luego, la velocidad para todo instante de tiempo es:

$$
\begin{aligned}
& \vec{v}(t)=20 \hat{\mathrm{i}}+(12,5-9,8 t) \hat{j}[m / s] \\
& \vec{v}(t)=\vec{v}_{x}(\mathrm{t}) \hat{\mathrm{i}}+\vec{v}_{y}(\mathrm{t}) \hat{j}[m / s]
\end{aligned}
$$

La posición para todo instante de tiempo está dada por:

$$
\begin{aligned}
& \left.\vec{r}(t)=\int \vec{v}(t) d t\right) \Rightarrow \vec{r}(t)=\int 20 \hat{\mathrm{i}}+(12,5-9,8 t) \hat{j} \bullet d t[m] \Rightarrow \\
& \vec{r}(t)=20 \hat{\mathrm{i}}+\left(12,5 \mathrm{t}-4,9 t^{2}\right) \hat{j}+c \Rightarrow t=0[s] \Rightarrow \vec{r}(0)=c=0[m] \\
& \therefore \vec{r}(t)=20 t \hat{\mathrm{i}}+\left(12,5 \mathrm{t}-4,9 t^{2}\right) \hat{j}[m] \\
& \Rightarrow \vec{r}(t)=\vec{r}_{x}(t) \hat{i}+\vec{r}_{y}(t) \hat{j}
\end{aligned}
$$

Condición final

$\vec{v}_{y}(t)=0$ (Punto máxima altura)

Luego el tiempo máximo que demora en alcanzar la altura máxima es:

$$
\begin{gathered}
0=(12,5-9,8 t) \Rightarrow t_{\text {máx }}=\frac{12,5}{9,8}=1,27[s] \\
\therefore \vec{r}_{y}(1,27)=\left(12,5 \bullet 1,27-4,9 \bullet(1,27)^{2}\right) \hat{j}[m]=7,97 \hat{j}[m]
\end{gathered}
$$

Luego $\vec{r}_{y}(1,27)=7,97 \widehat{j}[m]$ es altura máxima.

c) El alcance está dado por:

$\vec{r}_{x}(t)=20 t \hat{\mathrm{i}}$

Condición final

$$
\begin{aligned}
& \vec{r}_{y}(t)=5 \hat{j}[m] \wedge \vec{r}_{y}(t)=\left(12,5 \mathrm{t}-4,9 t^{2}\right) \hat{j}[m] \\
& \Rightarrow 5=\left(12,5 \mathrm{t}-4,9 t^{2}\right) \Rightarrow 4,9 t^{2}-12,5 \mathrm{t}+5=0 \\
& t_{1}=0,63[s] \uparrow(\text { subida }) \wedge t_{2}=2,05[s] \downarrow(\text { bajada })
\end{aligned}
$$

Los dos tiempos son solución, esto es, dos veces pasa por la misma altura, al subir y bajar.

$$
\begin{aligned}
& \vec{r}_{x}(t) \uparrow=20 \hat{t}=20 \bullet 0,63 \hat{\mathrm{i}}[\mathrm{m}]=12,6 \hat{\mathrm{i}}[\mathrm{m}] \\
& \vec{r}_{x}(t) \downarrow=20 \hat{\mathrm{i}}=20 \bullet 2,05 \hat{\mathrm{i}}[\mathrm{m}]=41 \hat{\mathrm{i}}[\mathrm{m}]
\end{aligned}
$$

La velocidad de la esquiadora al subir y bajar que pasa por la misma altura está dada por:

$$
\begin{aligned}
& \vec{v}(t)=20 \hat{i}+(12,5-9,8 t) \hat{j}[m / s] \\
& \vec{v}(t) \uparrow=20 \hat{i}+6,32 \hat{j}[m / s] \\
& \vec{v}(t) \downarrow=20 \hat{i}-7,59 \hat{j}[\mathrm{~m} / \mathrm{s}]
\end{aligned}
$$

